\title{
Size Control of Gold Nanoparticles Synthesized by Laser Ablation in Liquid Media
}

\author{
Daria Riabinina, ${ }^{1}$ Jianming Zhang, ${ }^{2}$ Mohamed Chaker, ${ }^{2}$ Joëlle Margot,, ${ }^{1}$ and Dongling Ma $^{2}$ \\ ${ }^{1}$ Département de Physique, Université de Montréal, C.P. 6128, Succursale Centre-Ville, Montréal, QC, Canada H3C 3J7 \\ ${ }^{2}$ Centre Énergie, Matériaux et Télécommunications, INRS, 1650 Lionel-Boulet, C.P. 1020, Varennes, Qc, Canada J3X 1S2
}

Correspondence should be addressed to Mohamed Chaker, chaker@emt.inrs.ca and Dongling Ma, ma@emt.inrs.ca

Received 9 November 2011; Accepted 7 December 2011

Academic Editors: R. B. Azevedo, L. A. Dykman, and M. Tommasini

Copyright ( 2012 Daria Riabinina et al. This is an open access article distributed under the Creative Commons Attribution License, which permits unrestricted use, distribution, and reproduction in any medium, provided the original work is properly cited.

\begin{abstract}
We investigate the influence of salt, acidic, and basic solutions (citrate, $\mathrm{NaOH}$, and $\mathrm{HCl}$ ) on the size of gold nanoparticles (AuNPs) synthesized by laser ablation in aqueous media. We found that NP size increases from $3 \mathrm{~nm}$ to $13 \mathrm{~nm}$ when Zeta potential varies from $-100 \mathrm{mV}$ to $-10 \mathrm{mV}$ whatever the concentration and the nature of chemical solution are namely, citrate, $\mathrm{NaOH}$, and $\mathrm{HCl}$. These results demonstrated that the final size of gold NPs produced by laser ablation in liquid media is mainly governed by the charge-dependent growth mechanism.
\end{abstract}

\section{Introduction}

Pulsed laser ablation in liquid media (PLAL) has become an increasingly important alternative approach for synthesis of colloidal suspensions with novel functional properties $[1,2]$. In particular, the fabrication of metallic nanoparticles (NPs) $[2,3]$, mostly gold and silver, has attracted much interest due to their surface plasmon resonance related properties that are potentially useful for their biological applications [4-7].

Numerous investigations have been performed in order to produce metallic NPs with controlled size and functional properties [8-11]. Variation of laser ablation parameters $[9,12]$ and chemical properties [13-16] of surrounding liquid media has been the primary focus of these studies. It has been demonstrated that the size of gold NPs produced in water increases with increasing laser pulse energy [17]. The variation in $\mathrm{pH}$ of various solutions influences not only the NP size $[13,18]$, but also the stability of gold colloids [19]. Despite numerous attempts to control the structural properties of gold NPs through synthesis parameters, a solid explanation of the influence of added chemicals on the size of gold NPs has not yet been proposed.

In this work, we investigate the structural properties of gold NPs synthesized in three different solutions (citrate,
$\mathrm{NaOH}$, and $\mathrm{HCl}$ ) in order to better understand the mechanism of NP growth during laser ablation in aqueous media.

\section{Experimental}

Gold NPs were prepared by pulsed laser ablation of a solid gold target placed at the bottom of a glass beaker filled with $3 \mathrm{~mL}$ of water or various concentrations of hydrochloric acid $(\mathrm{HCl})$, sodium hydroxide $(\mathrm{NaOH})$, and sodium citrate. A nanosecond $\mathrm{KrF}$ excimer laser (wavelength: $248 \mathrm{~nm}$, pulse duration: $17 \mathrm{~ns}$, repetition rate: $20 \mathrm{~Hz}$, laser fluence: $1 \mathrm{~J} / \mathrm{cm}^{2}$ ) was focused on the gold target with a $7.5 \mathrm{~cm}$ lens (spot size: $0.1 \mathrm{~cm}^{2}$ ). Several seconds of ablation generally lead to a red coloration of the solution which indicates the formation of gold NPs. Red color corresponds to absorption at $\sim 500 \mathrm{~nm}$ and is related to the surface plasmon resonance of freshly produced gold colloids.

Gold NPs were characterized by transmission electron microscopy (TEM) and a Zeta potential analyzer. TEM measurements were carried out to estimate the average size and size distribution of Au NPs. For TEM measurements, $\mathrm{Au}$ colloids were centrifuged on $\mathrm{Cu} / \mathrm{C}$ grids and analyzed using a Hitachi H-7100 TEM operated at $75 \mathrm{KeV}$. The Zeta potential of freshly prepared gold NPs was measured using a Brookhaven PALS Zeta Potential Analyzer. pH-values of 


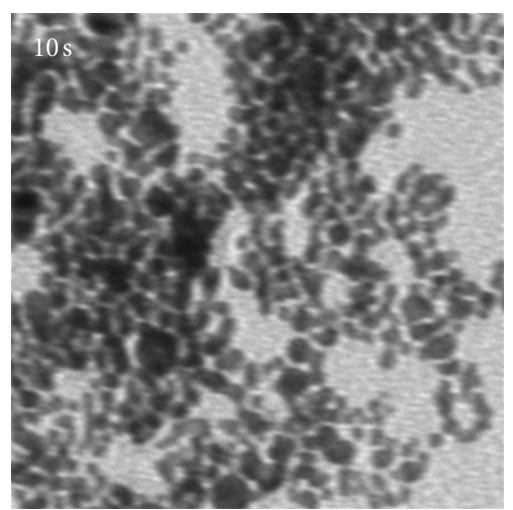

(a)

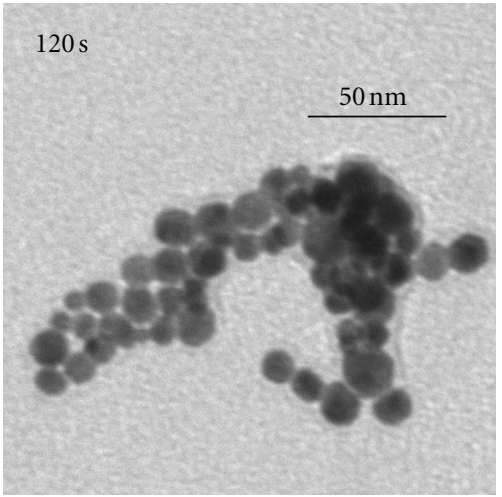

(b)

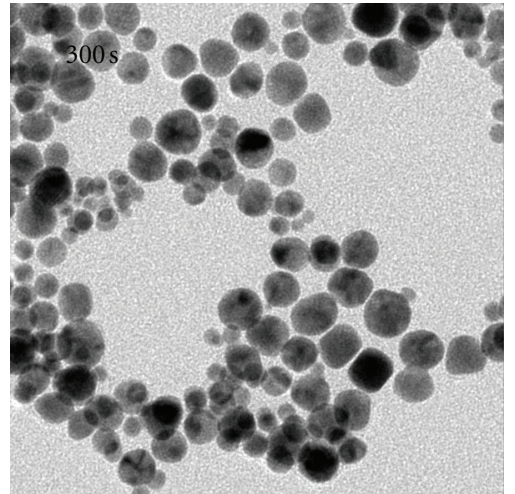

(c)

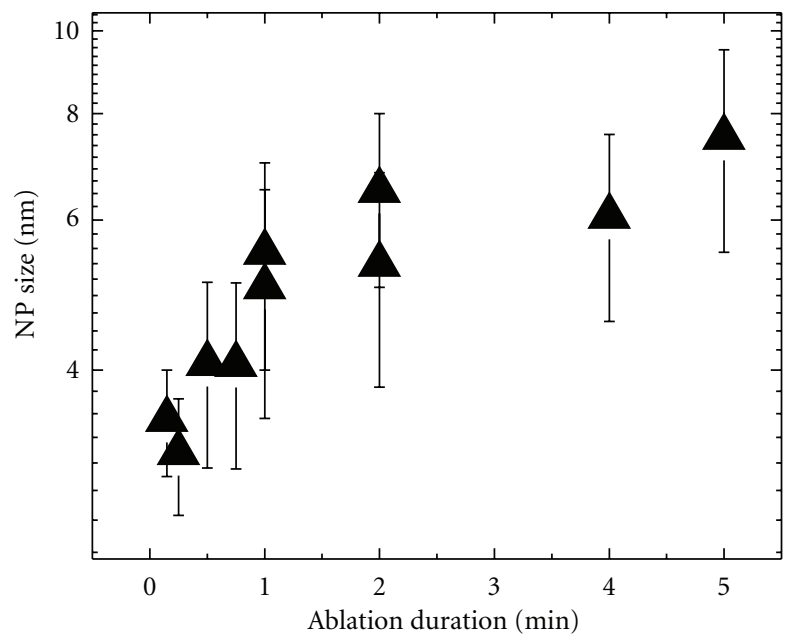

(d)

FIGURE 1: TEM images of gold NPs synthesized by pulsed laser ablation in $0.17 \%$ sodium citrate solution for different ablation durations, namely, (a) $10 \mathrm{sec}$, (b) $120 \mathrm{sec,} \mathrm{and} \mathrm{(c)} 300 \mathrm{sec}$ (laser wavelength $248 \mathrm{~nm}$, frequency $20 \mathrm{~Hz}$, pulse duration $17 \mathrm{~ns}$ ). (d) The size of gold NPs, extracted from TEM analysis, as a function of ablation duration.

colloidal gold solutions were measured using the MettlerToledo FG2 Portable pH meter.

\section{Results and Discussion}

Figures 1(a), 1(b), and 1(c) show TEM images of gold nanoparticles synthesized by laser ablation in $0.17 \%$ sodium citrate solution for different ablation durations. This solution is commonly used in chemical synthesis of gold NPs by citrate reduction method [20]. The average size of Au colloids as a function of ablation duration is plotted in Figure 1(d). The error bars correspond to the standard deviation of the average NP size. After $10 \mathrm{sec}$ of ablation, the presence of NPs of approximately $3 \mathrm{~nm}$ in diameter was observed, and after $5 \mathrm{~min}$ their average size increased up to about $6 \mathrm{~nm}$ in diameter. The observed increase in NP size as a function of ablation time indicates the presence of laser-nanoparticle interactions which yield to the fusion of colloids under laser irradiation.

In chemical growth of colloids, NP size can be controlled by adding molecules which act as stabilizing ligands at the freshly formed NP surface and thus quench its consequent growth. For example, in case of chemical synthesis of gold NPs by citrate reduction of auric acid, citrate molecules with negatively charged $-\mathrm{COO}^{-}$groups cover the gold colloid, thus creating an NP with a highly negative-charged surface [20]. The surface charge of these NPs can be generally quantified by Zeta potential measurements which give an indication of the NP surface charge due to the binding of the ligands to the gold surface.

In order to better understand the evolution of NP size as a function of ablation duration, we investigated the evolution of Zeta potential of gold NPs as a function of NP size. Figure 2 shows NP diameter as a function of Zeta potential value for gold NPs produced by PLAL in $0.17 \%$ sodium citrate solution, for ablation duration from $15 \mathrm{sec}$ to $360 \mathrm{sec}$. The variation in size of gold NPs is relatively small, while a significant increase of Zeta potential from $-42 \mathrm{mV}$ to $-89 \mathrm{mV}$ is observed with increasing ablation duration. This noticeable increase in Zeta potential value during laser ablation of gold in sodium citrate solution indicates a strong interaction between freshly synthesized gold NPs and the surrounding solution. This result suggests that citrate molecules actively bond to the Au surface, similar 


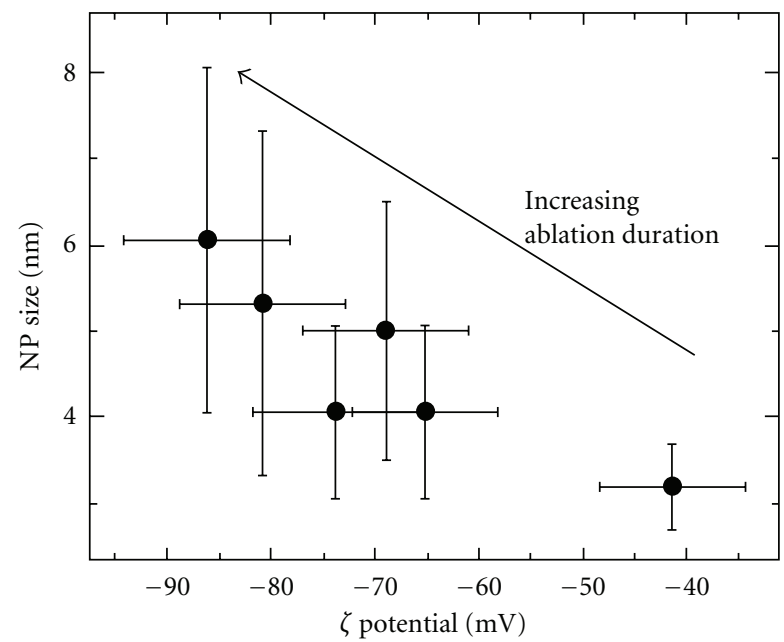

FIGURE 2: NP size as a function of Zeta potential for colloidal gold solutions synthesized by laser ablation in $0.17 \%$ sodium citrate solution for ablation duration varied from $15 \mathrm{sec}$ to $360 \mathrm{sec}$.

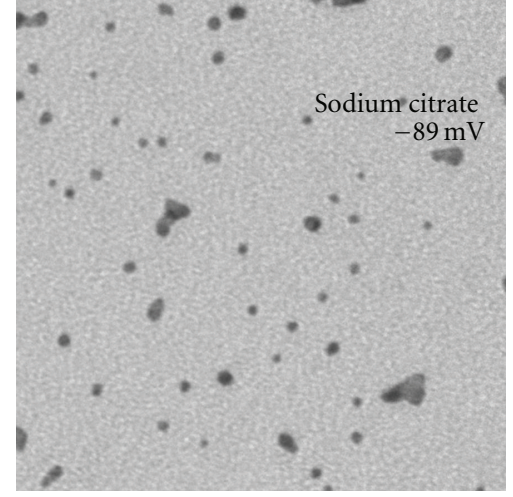

(a)

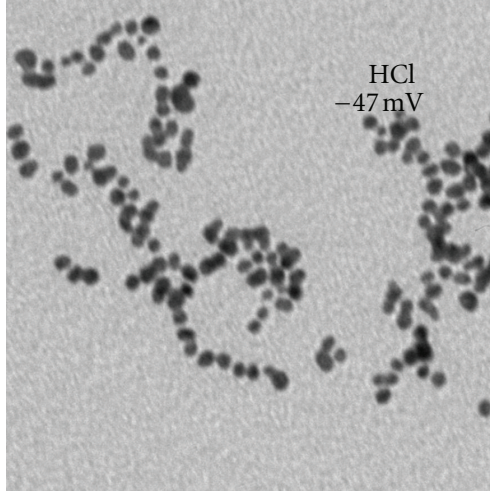

(b)

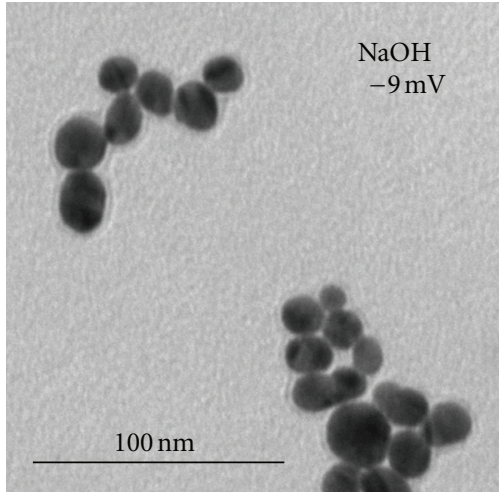

(c)

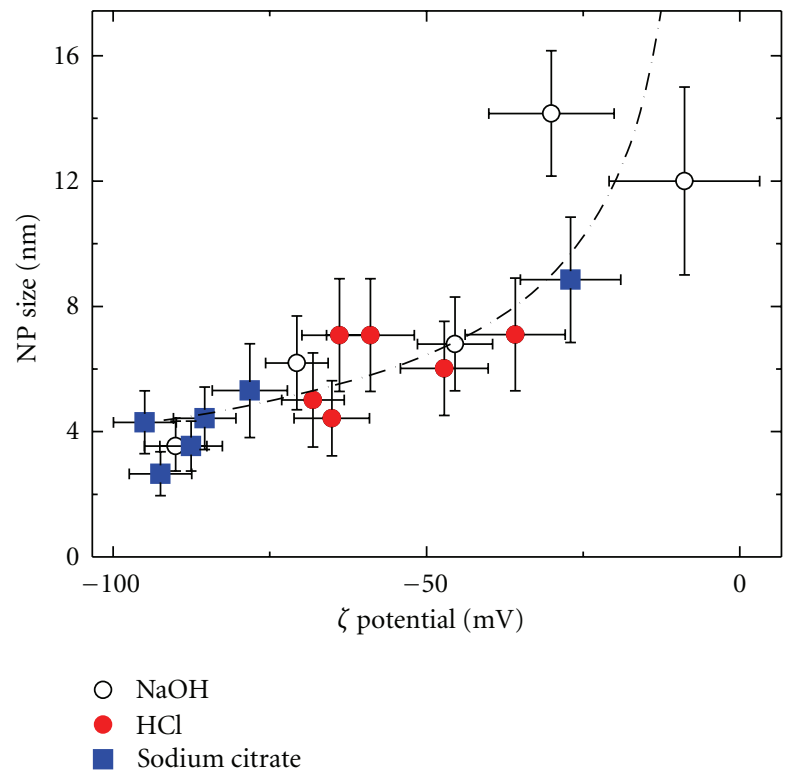

(d)

FIGURE 3: NP size as a function of Zeta potential for gold NPs synthesized by laser ablation in $\mathrm{NaOH}, \mathrm{HCl}$, and sodium citrate solutions of various concentrations (ablation duration of $60 \mathrm{sec}$ ). 
to that found for gold colloids prepared chemically by citrate reduction method. It is known that the Zeta potential of colloids may depend on the $\mathrm{pH}$ of the solution [19]. In order to understand whether the increase in Zeta potential value with increasing ablation duration of the gold NPs is due to the variation in $\mathrm{pH}$ of the solutions, we measured the $\mathrm{pH}$ for colloidal gold produced in sodium citrate solutions for different ablation durations. We observed that the increasing Zeta potential is accompanied by a slight increase in the $\mathrm{pH}$ value from 7.6 to 8.05 . However, as all the observed $\mathrm{pH}$ values are well above the three $\mathrm{p} K_{a}$ values of citric acid, this $\mathrm{pH}$ variation cannot absolutely explain the observed significant increase in Zeta potential from $-42 \mathrm{mV}$ to $-89 \mathrm{mV}$. Therefore, we conclude that the variation in Zeta potential is not due to the variation of $\mathrm{pH}$ value of solutions, but most likely it is associated with the surface chemistry modification of the produced gold NPs.

The important increase in Zeta potential of gold NPs with increasing ablation duration suggests the increase of the negative charge on NP surface. This surface modification certainly contributes to the repulsion between NPs and slows down the NP fusion, as confirmed by the size saturation at about $6 \mathrm{~nm}$ shown in Figure 1(d). These results suggest that the size of colloids synthesized by laser ablation in liquid media is governed by the charge-dependent process of NP fusion. The surface chemistry modification of NPs during laser ablation is likely to highly depend on surrounding solution.

To confirm this hypothesis on the influence of surrounding solution on NP size, we synthesized gold NPs in different solutions containing varied concentrations of sodium citrate, $\mathrm{NaOH}$, and $\mathrm{HCl}$. Figure 3 shows the $\mathrm{NP}$ size as a function of the Zeta potential of the gold NPs. The ablation duration was fixed at $60 \mathrm{sec}$ for all investigated samples in order to compare the size of NPs in solutions with the same total ablated mass of the gold. TEM images reveal the size and morphology of gold NPs produced in different solutions in which they exhibit various Zeta potential values. The NP size was found to be equal to about 2-5 nm for Zeta potential values greater than $-70 \mathrm{mV}$, and it gradually increased up to $13 \mathrm{~nm}$ for Zeta potential values of about $-10 \mathrm{mV}$. This result confirms that the growth of NPs produced by pulsed laser ablation in solutions is governed by a charge-dependent mechanism, and the NP size can be simply controlled by the type and concentration of chemical species in the surrounding solution. Moreover, this plot is particularly remarkable because all the experimental data are located on the same curve which indicates that this mechanism is universal for different solutions (acid, base, and salt solutions).

\section{Conclusion}

In summary, we investigated the influence of the presence of sodium citrate, $\mathrm{HCl}$, and $\mathrm{NaOH}$ on the size of gold NPs synthesized by laser ablation in aqueous media. We concluded that two main mechanisms influence the size of NPs. Firstly, the interaction between freshly ablated gold and surrounding solution determines the surface chemistry of produced gold NPs. Secondly, interactions between NPs under laser irradiation lead to fusion of gold colloids. The correlation between NP size and Zeta potential of colloidal gold showed that the size of gold NPs produced by laser ablation can be controlled by simple addition of various surfactants to the aqueous solution. These results demonstrated that the final size of gold NPs produced by laser ablation in liquid media is mainly governed by the charge-dependent growth mechanism.

\section{Acknowledgments}

The authors acknowledge funding from the Natural Sciences and Engineering Research Council of Canada and Fonds de la Recherche sur la Nature et les Technologies. D. Riabinina is grateful for financial support from FQRNT.

\section{References}

[1] V. Amendola, S. Polizzi, and M. Meneghetti, "Free silver nanoparticles synthesized by laser ablation in organic solvents and their easy functionalization," Langmuir, vol. 23, no. 12, pp. 6766-6770, 2007.

[2] G. Compagnini, A. A. Scalisi, and O. Puglisi, "Ablation of noble metals in liquids: a method to obtain nanoparticles in a thin polymeric film," Physical Chemistry Chemical Physics, vol. 4, no. 12, pp. 2787-2791, 2002.

[3] F. Mafuné, J. Kohno, Y. Takeda, and T. Kondow, "Full physical preparation of size-selected gold nanoparticles in solution: laser ablation and laser-induced size control," Journal of Physical Chemistry B, vol. 106, no. 31, pp. 7575-7577, 2002.

[4] X. M. Qian, X. H. Peng, D. O. Ansari et al., "In vivo tumor targeting and spectroscopic detection with surface-enhanced Raman nanoparticle tags," Nature Biotechnology, vol. 26, no. 1, pp. 83-90, 2008.

[5] H. He, C. Xie, and J. Ren, "Nonbleaching fluorescence of gold nanoparticles and its applications in cancer cell imaging," Analytical Chemistry, vol. 80, no. 15, pp. 5951-5957, 2008.

[6] E. Dulkeith, T. Niedereichholz, T. A. Klar et al., "Plasmon emission in photoexcited gold nanoparticles," Physical Review B, vol. 70, no. 20, Article ID 205424, 2004.

[7] G. Barbillon, J.-L. Bijeon, J. Plain, M. L. de la Chapelle, P. M. Adam, and P. Royer, "Biological and chemical gold nanosensors based on localized surface plasmon resonance," Gold Bulletin, vol. 40, no. 3, pp. 240-244, 2007.

[8] N. V. Tarasenko, A. V. Butsen, E. A. Nevar, and N. A. Savastenko, "Synthesis of nanosized particles during laser ablation of gold in water," Applied Surface Science, vol. 252, no. 13, pp. 4439-4444, 2006.

[9] J.-P. Sylvestre, A. V. Kabashin, E. Sacher, and M. Meunier, "Femtosecond laser ablation of gold in water: influence of the laser-produced plasma on the nanoparticle size distribution," Applied Physics A, vol. 80, no. 4, pp. 753-758, 2005.

[10] G. W. Yang, "Laser ablation in liquids: applications in the synthesis of nanocrystals," Progress in Materials Science, vol. 52, no. 4, pp. 648-698, 2007.

[11] A. Pyatenko, K. Shimokawa, M. Yamaguchi, O. Nishimura, and M. Suzuki, "Synthesis of silver nanoparticles by laser ablation in pure water," Applied Physics A, vol. 79, no. 4-6, pp. 803-806, 2004.

[12] P. Šmejkal, J. Pfleger, B. Vlčková, and O. Dammer, "Laser ablation of silver in aqueous ambient: effect of laser pulse 
wavelength and energy on efficiency of the process," Journal of Physics, vol. 59, no. 1, pp. 185-188, 2007.

[13] J. P. Sylvestre, A. V. Kabashin, E. Sacher, M. Meunier, and J. H. T. Luong, "Stabilization and size control of gold nanoparticles during laser ablation in aqueous cyclodextrins," Journal of the American Chemical Society, vol. 126, no. 23, pp. 7176-7177, 2004.

[14] R. M. Tilaki, A. Irajizad, and S. M. Mahdavi, "Stability, size and optical properties of silver nanoparticles prepared by laser ablation in different carrier media," Applied Physics A, vol. 84, no. 1-2, pp. 215-219, 2006.

[15] K. Sišková, B. Vlčková, P.-Y. Turpin, C. Fayet, J. Hromádková, and M. Slouf, "Effect of citrate ions on laser ablation of Ag foil in aqueous medium," Journal of Physics, vol. 59, no. 1, article no. 044, pp. 202-205, 2007.

[16] G. Bajaj and R. K. Soni, "Effect of liquid medium on size and shape of nanoparticles prepared by pulsed laser ablation of tin," Applied Physics A, vol. 97, no. 2, pp. 481-487, 2009.

[17] S. Besner, A. V. Kabashin, F. M. Winnik, and M. Meunier, "Ultrafast laser based "green" synthesis of non-toxic nanoparticles in aqueous solutions," Applied Physics A, vol. 93, no. 4, pp. 955-959, 2008.

[18] A. V. Kabashin, M. Meunier, C. Kingston, and J. H. T. Luong, "Fabrication and characterization of gold nanoparticles by femtosecond laser ablation in an aqueous solution of cyclodextrins," Journal of Physical Chemistry B, vol. 107, no. 19, pp. 4527-4531, 2003.

[19] J.-P. Sylvestre, S. Poulin, A. V. Kabashin, E. Sacher, M. Meunier, and J. H. T. Luong, "Surface chemistry of gold nanoparticles produced by laser ablation in aqueous media," Journal of Physical Chemistry B, vol. 108, no. 43, pp. 16864-16869, 2004.

[20] G. Frens, "Controlled nucleation for the regulation of the particle size in monodisperse gold suspensions," Nature Physics, vol. 241, pp. 20-22, 1973. 

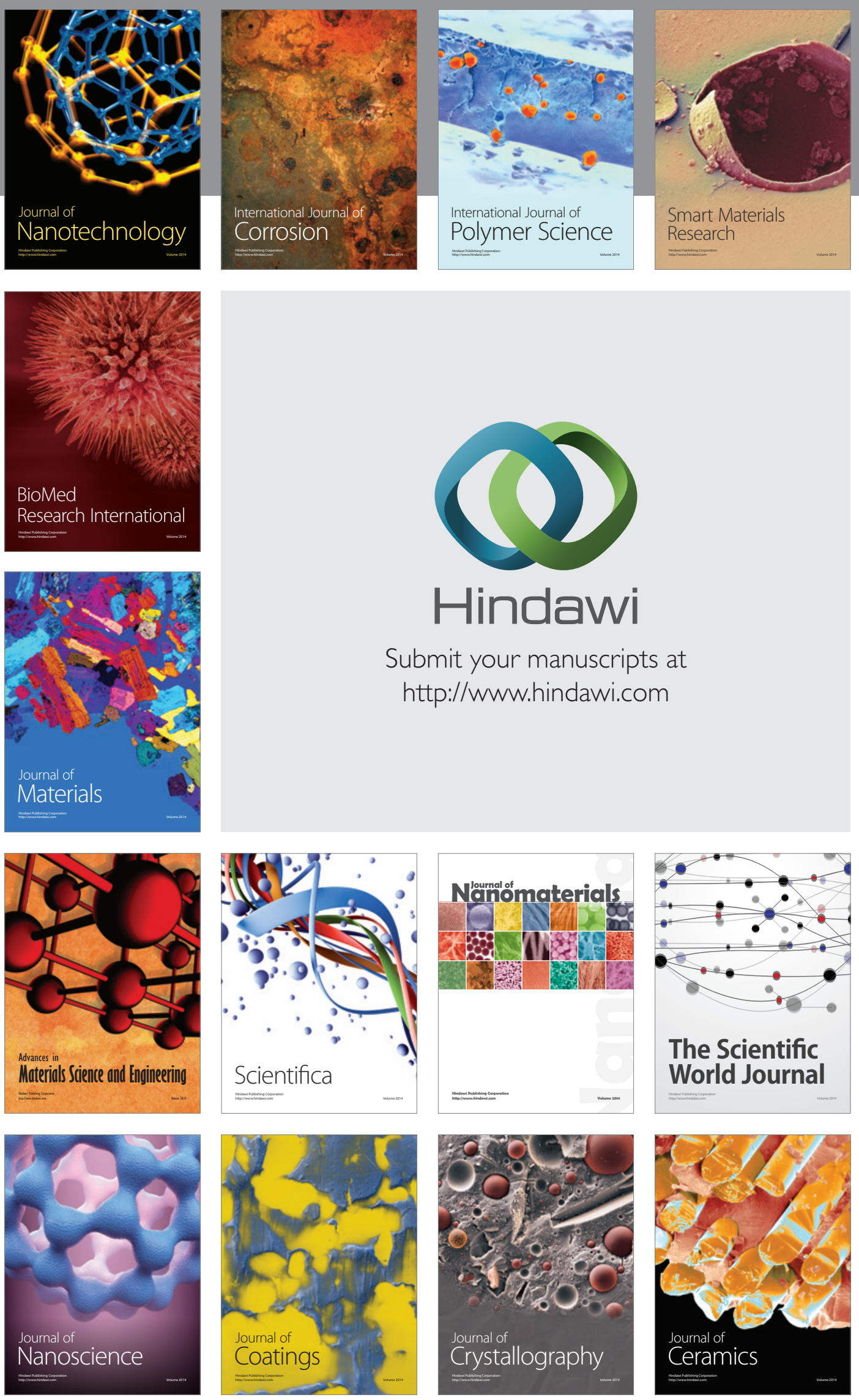

The Scientific World Journal

Submit your manuscripts at

http://www.hindawi.com

\section{World Journal}

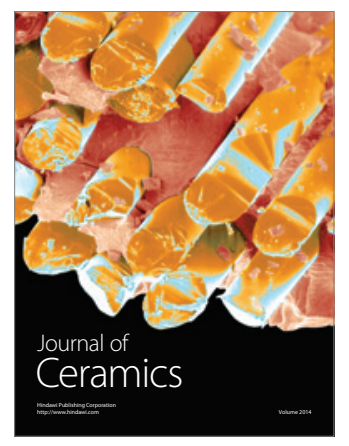

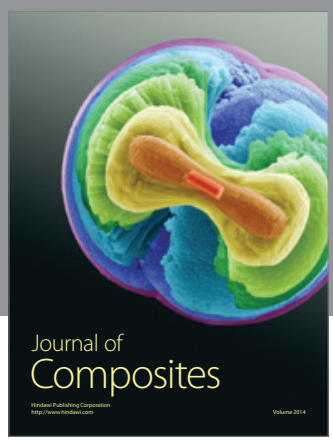
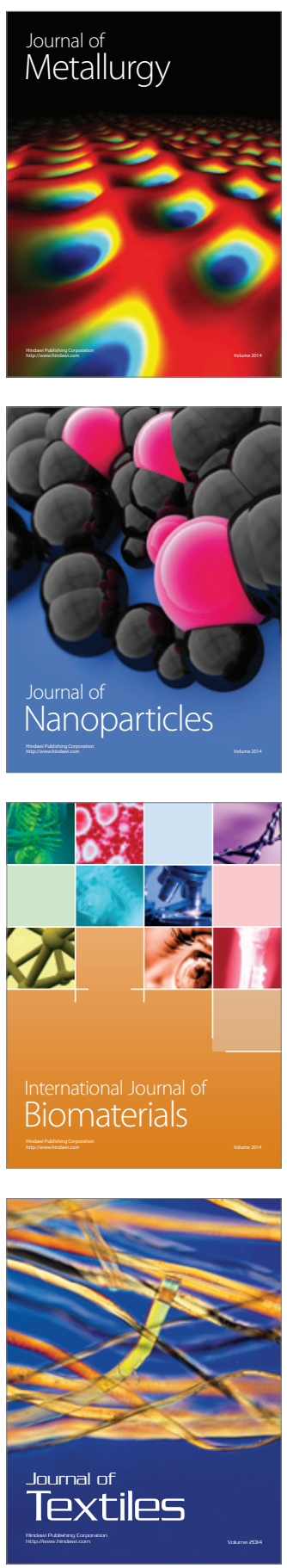